\title{
Dynamics of the cerebrospinal fluid and the spinal dura mater ${ }^{1}$
}

\author{
ALBERT N. MARTINS, JOHN K. WILEY, AND PAUL W. MYERS \\ From the Neurosurgery Service, Wilford Hall U.S.A.F. Hospital, San Antonio, Texas, U.S.A.
}

SUMMARY During myelography we observed the contrast material in the spinal subarachnoid space while we changed: (1) the intracranial blood volume by $\mathrm{CO}_{2}$ inhalation, hyperventilation, and jugular vein compression; (2) the intra-abdominal and intrathoracic pressure by forced expiration with glottis closed; and (3) the CSF volume by withdrawals and reinjections of fluid. The spinal dural sac enlarges with increases in volume of both intracranial blood and CSF. It partially collapses ${ }_{\rightarrow}^{\text {s }}$ with reductions in volume of both intracranial blood and CSF. With increases in intra-abdominal. and intrathoracic pressure, the thoracolumbar sac partially collapses, while the cervical sac enlarges. $\vec{\omega}$ From these observations we conclude that the spinal dural sac is a dynamic structure, readily 2 changing its capacity in response to prevailing pressure gradients across its walls. It acts as a reservoir for CSF, which moves to and fro through the foramen magnum in response to changes in cerebral ${ }_{i}^{\circ}$ blood flow. By its bladder-like ability to alter its capacity, the spinal dural sac provides the 'elasticity' of the covering of the central nervous system.

By additions and withdrawals of fluid, we can quite easily change within limits the volume of the pool of cerebrospinal fluid (CSF) in which are immersed the brain and spinal cord. Consequently, the covering of the central nervous system (CNS) cannot be regarded as truly rigidthat is, it possesses, for want of a better term, 'elasticity'. What provides this so-called elasticity? Certainly not the cranial dura mater, for, with a closely applied rigid bony skull as a buttress, the cranial dura mater and the skull form together a container of unalterable capacity. On the contrary, the spinal dura mater (together with the arachnoid mater) buttressed not by bone but by a network of veins and fat in the epidural space seems potentially able to alter its capacity.

Our current concepts of dural elasticity, however, remain influenced by the work of Weed and his co-workers, completed almost 40 years ago (Weed, 1929; Weed and Flexner, 1933; Weed, Flexner, and Clark, 1932). Although recognizing that the spinal dura mater has potential elasticity, they regarded the role of the venous system of the CNS as preeminent in providing for the elasticity of the CNS covering. After a series of animal experiments concerned with

\footnotetext{
1 Presented in part as a movie to the meeting of The American Association of Neurological Surgeons, Houston, April 1971.
}

CSF pressure changes produced by changes position, Weed and his co-workers concluded that the apparent elasticity of the dura mater $\$ \Phi^{\circ}$ basically provided by the changes in volume $\stackrel{\text { ? }}{\rightarrow}$ the intradural venous blood (Weed et al., 193\% $\overrightarrow{0}$ and that induced changes in intracranial CSF N volume were possible chiefly because the 'cerebral venous bed' compensated for them (Weed and Flexner, 1933). Similarly, Ryder, Espey, Kimbell, Penka, Rosenauer, Podolsky, and Evans (1953) concluded that 'changing the volume of the cerebrospinal fluid space induces $\overrightarrow{\vec{O}}$ an immediate reciprocal change in the cranio- $\exists$ spinal blood volume in all men'. In 1960疍 Bowsher discarded completely the concept of the dura mater being elastic when he wrote '... the dura should be regarded as a rigid container . . . the elasticity of the fluid system is due mainly to the elasticity of its contained veins'.

But to the myelographer and the surgeon alike, the spinal dural sac does not appear to beo rigid at all. Indeed, according to Pollock and Boshes (1936), as long ago as 1892 H. Grashey을 recognized that the spinal dural sac probablyn could alter its capacity. Subsequently others also have implied agreement (Foldes, Keutmann, $N$ and Hunt, 1958; Kety, 1965; DuBoulay and $\tilde{\omega}$ El Gammal, 1966; Ponssen and Van Den Bos, 1971). Nevertheless, some authors continue to 
express or imply acceptance of the concept of dural elasticity as advocated by Weed's and Ryder's groups (Von Storch, Carmichael, and Banks, 1937; Kunkle, Ray, and Wolff, 1943; Hayden, Shurtleff, and Foltz, 1970; Katzman and Hussey, 1970; Sibayan, Begeman, King, Gurdjian, and Thomas, 1970). Therefore we felt it would be profitable to re-examine what the spinal dural sac contributes to this elasticity. To this end we studied and report in this paper what happens to a Pantopaque-filled spinal dural sac during routine clinical myelography when one changes: (1) the intracranial blood volume by $\mathrm{CO}_{2}$ inhalation, hyperventilation, and jugular compression; (2) the intra-abdominal and intrathoracic pressure by forced expiration with glottis closed (Valsalva manoeuvre); and (3) CSF volume by withdrawals and re-injections of fluid.

\section{METHOD}

Twelve informed volunteers were selected from among those patients who were to undergo myelography for suspected intervertebral disc disease. The lumbar puncture was accomplished; about $12 \mathrm{ml}$. CSF was withdrawn and an equal amount of Pantopaque (iophendylate) injected intrathecally. After the diagnostic procedure was concluded routinely, the following manoeuvres were performed while the changes in the column of Pantopaque were observed fluoroscopically and recorded both on regular radiographs and cineradiographically: with the subjects horizontal and Pantopaque filling his cervical canal from the rim of the foramen magnum to the fifth or sixth cervical vertebra, he was asked to perform a 'Valsalva' two or three times. The column of contrast medium was returned to the lumbosacral sac, the subject positioned horizontally, and then he was asked in turn to hyperventilate for two minutes, breathe $10 \% \mathrm{CO}_{2}$ in oxygen for two minutes, and repeat the 'Valsalva' two or three times. Then both of his jugular veins were compressed for about 15 seconds. Finally $15 \mathrm{ml}$. CSF was removed and then re-injected.

\section{RESULTS}

During hyperventilation the sac collapses partially as indicated by the Pantopaque column narrowing and elongating. When hyperventilation stops the sac gradually resumes its original appearance within two minutes.

When $10 \% \mathrm{CO}_{2}$ in oxygen is inhaled, the sac distends as indicated by a column that widens as it moves caudally, filling the lumbosacral nerve roots. The sac regains its original appearance within two minutes after $\mathrm{CO}_{2}$ inhalation is stopped.

In response to forced expiration with glottis closed (Valsalva manoeuvre), the column in the lumbosacral area almost instantaneously narrows and elongates as the sac collapses partially. In the cervical area, with the Pantopaque column positioned at the foramen magnum, the sac abruptly widens slightly during the manoeuvre. The column does not move intracranially. At the end of the Valsalva manoeuvre, the column in either location returns quickly to its previous appearance and position.

In response to bilateral jugular compression, the sac again distends as indicated by the column that widens, moves caudally and fills the lumbosacral nerve root sleeves. The response reaches maximum in five to 10 seconds. With release of jugular compression, the column regains in a few seconds its pre-compression appearance.

When CSF is withdrawn, the sac partially collapses as indicated by the Pantopaque column becoming narrower and longer; when CSF is re-injected, the sac recovers its former configuration.

The changes in appearance and position of the Pantopaque column were the same in all subjects for each manoeuvre, although their magnitude did vary. The changes are somewhat difficult to document satisfactorily with ordinary serial radiographs, but are easy to appreciate at fluoroscopy as well as upon subsequent study of the cineradiographs (Figure).

\section{DISCUSSION}

In interpreting the results of these experiments, we have assumed that changes in the appearance of the column of Pantopaque reflect changes in the size, and hence capacity, of the visualized segment of the spinal dural sac. When the silhouette of a $12 \mathrm{ml}$. column of Pantopaque becomes longer and narrower, we have assumed that the sac in that area has partially collapsed. When it becomes shorter and wider, we have conversely assumed that the sac there has distended and increased its capacity. If our assumptions are valid, we may conclude from these simple and easily reproducible experiments that the spinal dural sac is a dynamic structure that readily changes its capacity in response to prevailing pressure gradients across its walls. And since the cranial and spinal CSF compartments normally communicate freely at the foramen 


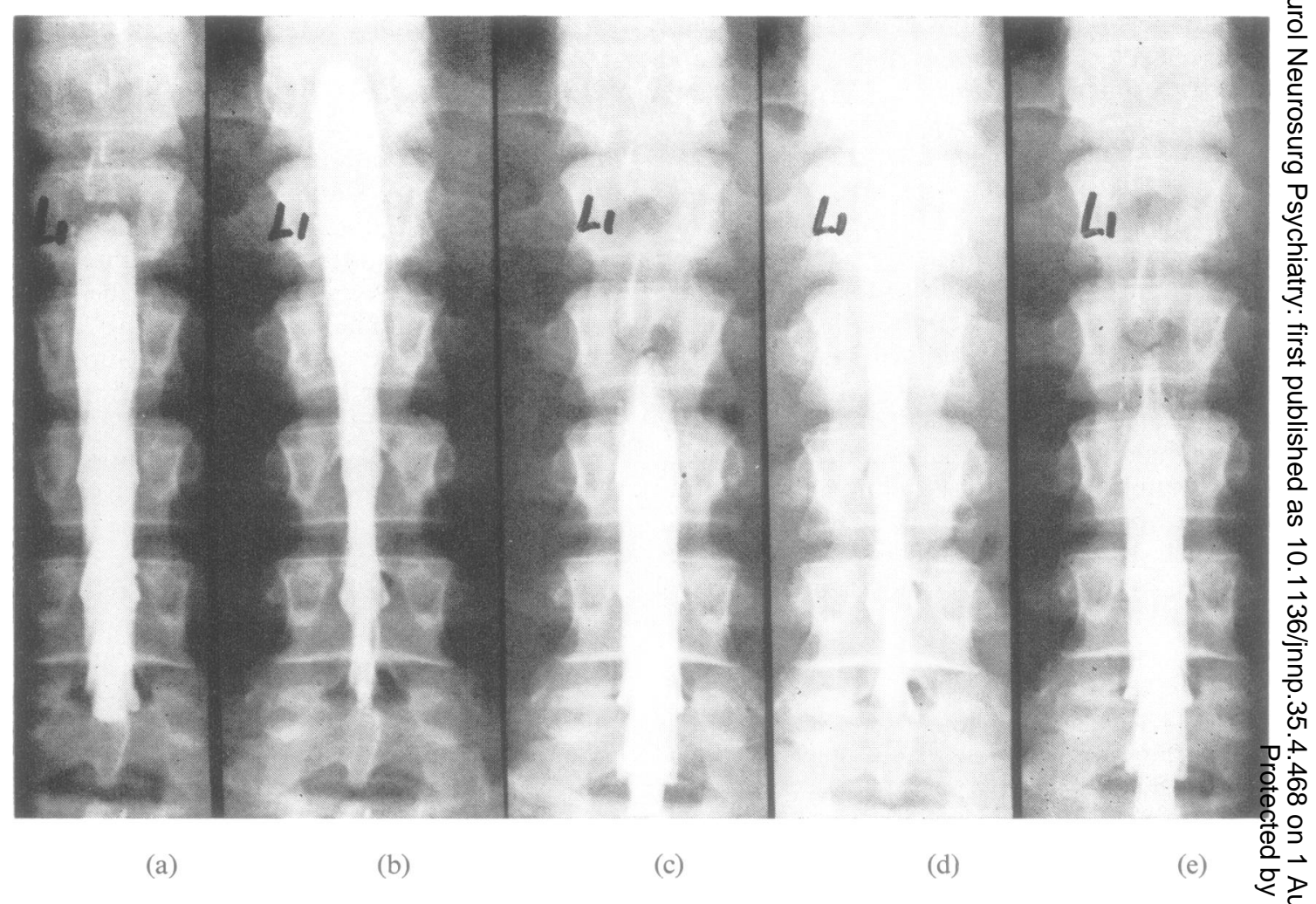

FIG. 1. Serial radiographs of a Pantopaque-filled lumbosacral spinal dural sac from a single subject. Between exposures the horizontal position of the subject as well as the position of the $\mathrm{x}$-ray tube and cassette hold $\frac{\text { of }}{+}$ remain unchanged. In response to changes in both intracranial blood volume and pressure within the thorax an $\overrightarrow{0}$ abdomen induced by various manoeuvres, the spinal dural sac readily alters its volume and returns to the bas $\overrightarrow{\vec{t}} \underset{\mathrm{N}}{\overrightarrow{\mathrm{d}}}$ appearance at the end of each manoeuvre. From left to right: (a) basal appearance; (b) appearance after two minutes of hyperventilation; (c) appearance after breathing $10 \% \mathrm{CO}_{2}$ in oxygen for two minutes; (d) appearance during Valsalva manoeuvre; (e) appearance after bilateral jugular compression for 15 seconds. (On cinéradiographs the changes are more readily appreciated.)

magnum, it follows that a spinal dural sac of variable capacity can account for the limited elasticity of the entire covering of the CNS.

Other data, aside from our own, conflict with a hypothesis that maintains that the apparent elasticity of the dura mater is really an attribute of its contained veins, which are presumed to expand or collapse in response to intradural volume and pressure changes. Specifically, such an hypothesis could not explain the intradural volume adjustments dictated by rapid changes in CNS blood volume known to accompany changes in cerebral blood flow (Risberg, Ancri, and Ingvar, 1969). With these changes of CNS blood volume, the dural envelope must likewise change its capacity. According to current evidence, neither brain parenchyma nor the CSF compartment is able to change its own volume reciprocally and quickly enough in response to changes in cerebral blood flow to maintain constant the total volume enclosed within the dura mater (McIlwain, 1966; Rubin, Henderson, Ommaya, Walker, and Rall, 1966; Davson, 1967; Cutler, Page, Galicich, and Watters, 1968). Furthermore, many independent investigators, Cushing himself the first, have observed directly 을 that cerebral veins do not collapse significantly when CSF pressure is increased by intrathecal injection of fluid (Cushing, 1902; Wolff and Forbes, 1928; Fog, 1933; Wright, 1938; Hekmatpanah, 1970). Described first by Wright ${ }_{N}$ (1938), the 'cuff-constriction' of the bridging $\omega$ veins at their junction with the dural venous sinuses, which varies in degree according to the 
CSF pressure, apparently prevents collapse of the veins. This phenomenon is governed by the principles of fluid dynamics related to flow through collapsible tubes that are surrounded by fluid at higher pressure than the outflow pressure, as elucidated by Permutt and Riley (1963).

Accordingly, the theoretical and experimental evidence mentioned above, together with our own observations, support an alternative view. The elasticity of the dura mater we believe can best be explained by the collapsible nature of the spinal dural sac, which we envision as an elongated thin-walled bladder, normally not filled to capacity, giving to or accepting from the intracranial spaces, via the foramen magnum, CSF according to the prevailing pressure gradient. In changing its capacity, true rubber balloon-like dural elasticity probably plays an insignificant role. Rather the sac seems to fill and empty like a cloth or leather water bag. Simply by removing and reinjecting CSF and observing the changes in the appearance of the column of Pantopaque, one readily establishes that the spinal dural sac can change its capacity. One adds further evidence of a more physiological nature by observing the sac as the intracranial blood volume is increased either by hypercapniainduced increases of cerebral blood flow or by the intracranial venous congestion that follows jugular compression. The caudal sac, silhouetted by the Pantopaque column, is seen to enlarge first and then return to its previous appearance when either manoeuvre is terminated, pari passu with return of intracranial blood volume to normal. As the intracranial blood volume falls in response to hypocapnia induced by hyperventilation, the sac partially collapses as CSF leaves it to replace the volume of blood that left the cranial cavity. This apparent reciprocal relationship between the intracranial blood and CSF compartments, permitted by the spinal dural sac, allows the brain both the protection of the rigid skull and the flexibility of a vascular bed that can alter its capacity like that of any other organ. In this generally ignored role the CSF must certainly perform one of its important functions.

Because the spinal canal is rigid, we must postulate that with each change in the volume of the spinal dural sac, the volume of tissue within the canal, but extradural, must also change by an equal and opposite amount. Since loose fatty tissue and a capacious venous plexus occupy the spinal epidural space, one or both of these elements must be involved in the volume adjustments. Indeed, one might argue that the changes observed in the sac during hyperventilation and $\mathrm{CO}_{2}$ inhalation are initiated by changes within the spinal epidural compartment; but there is little physiological evidence to support such an assumption. Evidence favours the wellknown alterations in cerebral blood flow, which accompany changes in blood $\mathrm{CO}_{2}$ tension, as the factors that alter CSF pressure and in turn alter the size of the sac (Lassen and Ingvar, 1963; Harper, 1965; Meyer and Gilroy, 1968).

While the amount of epidural fat does seem capable of change by sliding into and out of the spinal canal through the neural foramina, the venous plexus seems the more likely element to play the important role. A picture thus emerges of the spinal dural sac acting in reciprocal concert with the epidural venous blood volume to provide the limited but definite elasticity of the dura mater.

This reciprocal relationship between the CSFfilled sac and the epidural venous plexus can explain also the exquisite sensitivity of the CSF pressure to sudden changes in intrathoracic and intra-abdominal pressure, which we observe during the Valsalva manoeuvre, coughing, straining, and during the respiratory cycle as well. Observing a column of Pantopaque during lumbar myelography, we note a sudden narrowing and elongation of the column as the sac partially collapses almost immediately in response to a Valsalva manoeuvre, retaining this appearance until the manoeuvre is ended. Judging from the rates of change observed during myelography, as well as from the rapidity with which CSF pressure is known to rise in response to the Valsalva manoeuvre, we conclude that CSF pressure (and intracranial pressure as well) becomes essentially equal to the pressure within the thorax and abdomen in a matter of a second or less. To account for this partial collapse of the thoracolumbar sac, filled as it is with incompressible tissue and CSF, we assume that with the rise in pressure within the thorax and abdomen, venous blood is forced rapidly from the vena cava and other large veins within the body cavities into the thoracolumbar epidural venous plexus. Thus, through these venous channels the intrathoracic and intra-abdominal pressures are transmitted to the collapsible dural sac to the spinal CSF, and in turn intracranially throughout the CSF compartment, consonant with the principles of hydraulics. But since the sac does 
partially collapse, some CSF must be temporarily displaced. Our observations of a Pantopaque column in the cervical canal poised at the rim of the foramen magnum suggest that, in response to the Valsalva manoeuvre, CSF flows into the cervical subarachnoid space because the column there widens slightly. Furthermore, since the column of Pantopaque does not move intracranially through the foramen magnum, we assume that CSF is not forced into the rigid cranial vault by a Valsalva manoeuvre. These observations lend support to the view that intracranial veins do not collapse in response to induced changes in CSF pressure. For if they did collapse, we assume that CSF would have moved intracranially and with it the column of Pantopaque.

Since the cervical dural sac is limited in how much it can distend by the closely applied bony spine, it very rapidly becomes filled to capacity. At this moment, when the pressure within the thorax and abdomen cannot reduce further the size of the thoracolumbar sac by dislocating fluid into the cervical canal, pressures within the thorax and abdomen, as well as throughout the entire CSF compartment, become essentially equal, all taking place almost instantaneously. We assume that because both the epidural venous plexus and the dural sac in the cervical region are not directly exposed to pressures developed within the body cavities, each can vary its volume independently of the thoracolumbar epidural venous volume.

Prolonged Valsalva manoeuvre or straining impedes venous return. But the contribution of intracranial venous congestion, which attends this venous obstruction, is late in evolving compared with the dramatic and abrupt rise early in the manoeuvre that derives from the dynamics just mentioned.

In applying these concepts to the clinical situation, we would assume for example that the bulge of a crying infant's fontanelle is not caused by venous congestion but by CSF being forced to flow from the thoracolumbar sac into the non-rigid skull by the increased intrathoracic and intra-abdominal pressure. We may apply these concepts also to an understanding of the events surrounding the rupture of a weakened intracranial vessel concurrent with an episode of coughing or straining. There is general belief that the catastrophe is related in time to the sudden rapid rise in intracranial pressure. However, if the dynamics are as presented here, the intracranial vessel would be more prone to rupture after the cough or strain, when the CSF pressure suddenly falls. Because initially, as the $\stackrel{\mathbb{D}}{=}$ intrathoracic and intra-abdominal pressure is transmitted to the spinal CSF and thence intra- $\frac{\widehat{A}}{0}$ cranially, the pressure gradient from the intravascular blood to the extravascular CSF across the blood vessel wall falls, thereby reducing the transmural pressure gradient $\stackrel{0}{3}$ favouring vessel rupture. Subsequently, when the episode is over and the intracranial CSF pressure $\stackrel{\overrightarrow{\vec{S}}}{\vec{A}}$ falls sharply, this transmural pressure gradient $\overline{0}$ favouring rupture returns to its former resting level, or perhaps even surpasses it. One may $\frac{\bar{s}}{7}$ speculate that at the end of an episode of cough- $\mathbb{\AA}$ ing or straining, as the intracranial pressure is falls, the autoregulatory mechanism concerned $\overrightarrow{0}$ with maintaining a normal rate of cerebral blood flow while the intracranial pressure is high will not reverse itself with equal speed. For this instant, flow through the weakened vessel will be greater than normal, and the transmural pressure of gradient favouring rupture will be also greates than normal.

\section{CONCLUSIONS}

The spinal dural sac acts as a reservoir for CSE readily changing its capacity in response pressure gradients developed within the CSF ant 6 the spinal epidural venous plexus. The main ? implications of this conclusion are four:

1. The elasticity of the dura mater, demonstrated when one artificially changes CSF volume with fluid additions and withdrawals, is a function of the spinal dural sac rather than the intradural cerebrospinal venous vascular bed.

2 . Because it can readily alter its capacity within limits, the spinal dural sac plays a primary role in the dynamics of CSF pressure changes that accompany changes in position.

3. The spinal dural sac, by acting as $a \frac{0}{0}$ reservoir for CSF, permits the vascular bed of 3 the brain to alter its total volume in response to $\delta$ changes in cerebral blood flow.

4. The sensitivity of the CSF pressure to changes in intrathoracic and intra-abdominal pressure depends upon the relationship of the epidural venous plexus to the spinal dural sac.

\section{REFERENCES}

Bowsher, D. (1960). Cerebrospinal Fluid Dynamics in Health and Disease. Thomas: Springfield, Ill. 
Cushing, H. (1902). Some experimental and clinical observations concerning states of increased intracranial tension. American Journal of Medical Sciences, 124, 375-400.

Cutler, R. W. P., Page, L., Galicich, J., and Watters, G. V. (1968). Formation and absorption of cerebrospinal fluid in man. Brain, 91, 707-720.

Davson, H. (1967). Physiology of the Cerebrospinal Fluid. Churchill: London.

Du Boulay, G. H., and El Gammal, T. (1966). The classification, clinical value and mechanism of sella turcica changes in raised intracranial pressure. British Journal of Radiology, 39, 422-442.

Fog, M. (1933). Influence of intracranial hypertension upon the cerebral circulation. Acta Psychiatrica (Kbh.), 8, 191198.

Foldes, F. F., Keutmann, E., and Hunt, R. D. (1958). The effect of continuous removal of cerebrospinal fluid on cerebrospinal fluid pressure. Anaesthesist, 7, 261-265.

Harper, A. M. (1965). Physiology of cerebral bloodflow. British Journal of Anaesthia, 37, 225-235.

Hayden, P. W., Shurtleff, D. B., and Foltz, E. L. (1970). Ventricular fluid pressure recordings in hydrocephalic patients. Archives of Neurology, 23, 147-154.

Hekmatpanah, J. (1970). Cerebral circulation and perfusion in experimental increased intracranial pressure. Journal of Neurosurgery, 32, 21-29.

Katzman, R., and Hussey, F. (1970). A simple constantinfusion manometric test for measurement of CSF absorption I. Rationale and method. Neurology (Minneapolis), 20, 534-544.

Kety, S. (1965). Remarks, in Cerebral Vascular Diseases. Transactions of the Fourth Conference, Princeton, N.J., 1964. Pp. 83. Edited by C. H. Millikan, R. G. Siekert, and J. P. Whisnant. Grune and Stratton: New York.

Kunkle, E. C., Ray, B. S., and Wolff, H. G. (1943). Experimental studies on headache: analysis of the headache associated with changes in intracranial pressure. Archives of Neurology and Psychiatry, 49, 323-358.

Lassen, N. A., and Ingvar, D. H. (1963). Regional cerebral blood flow measurement in man. Archives of Neurology, 9, 615-622.

Meyer, J. S., and Gilroy, J. (1968). Regulation and adjustment of the cerebral circulation. Diseases of the Chest, 53, 30-37.

McIlwain, H. (1966). Biochemistry and The Central Nervous System. 3rd edition. Pp. 412. Churchill: London.
Permutt, S., and Riley, R. L. (1963). Hemodynamics of collapsible vessels with tone: the vascular waterfall. Journal of Applied Physiology, 18, 924-932.

Pollock, L. J., and Boshes, B. (1936). Cerebrospinal fluid pressure. Archives of Neurology and Psychiatry, 36, 931974.

Ponssen, H., and Van Den Bos, G. C. (1971). Influence of the circulation on the CSF pressure wave. (Abstract.) Journal of Neurology, Neurosurgery, and Psychiatry, 34, 108.

Risberg, J., Ancri, D., and Ingvar, D. H. (1969). Correlation between cerebral blood volume and cerebral blood flow in the cat. Experimental Brain Research, 8, 321-326.

Rubin, R. C., Henderson, E. S., Ommaya, A. K., Walker, M. D., and Rall, D. P. (1966). The production of cerebrospinal fluid in man and its modification by acetazolamide. Journal of Neurosurgery, 25, 430-436.

Ryder, H. W., Espey, F. F., Kimbell, F. D., Penka, E. J., Rosenauer, A., Podolsky, B., and Evans, J. P. (1953). The mechanism of the change in cerebrospinal fluid pressure following an induced change in the volume of the fluid space. Journal of Laboratory and Clinical Medicine, 41, 428-435.

Sibayan, R. Q., Begeman, P. C., King, A. I., Gurdjian, E. S., and Thomas, L. M. (1970). Experimental hydrocephalus: ventricular cerebrospinal fluid pressure and waveform studies. Archives of Neurology, 23, 165-172.

Von Storch, T. J. C., Carmichael, E. A., and Banks, T. E. (1937). Factors producing lumbar cerebrospinal fluid pressure in man in the erect posture. Archives of Neurology and Psychiatry, 38, 1158-1175.

Weed, L. H. (1929). Some limitations of the Monro-Kellie hypothesis. Archives of Surgery, 18, 1049-1068.

Weed, L. H., and Flexner, L. B. (1933). The relations of the intracranial pressures. American Journal of Physiology, 105, 266-272.

Weed, L. H., Flexner, L. B., and Clark, J. H. (1932). Effect of dislocation of cerebrospinal fluid upon its pressure. American Journal of Physiology, 100, 246-261.

Wolff, H. G., and Forbes, H. S. (1928). The cerebral circulation: observations of the pial circulation during changes in intracranial pressure. Archives of Neurology and Psychiatry, 20, 1035-1047.

Wright, R. D. (1938). Experimental observations on increased intracranial pressure. Australian and New Zealand Journal of Surgery, 7, 215-235. 